\title{
Anisotropic Smoothing Regularization (AnSR) in Thirion's Demons Registration Evaluates Brain MRI Tissue Changes Post-Laser Ablation*
}

\author{
Eileen Hwuang ${ }^{1}$, Shabbar Danish ${ }^{3}$, Mirabela Rusu ${ }^{2}$, Rachel Sparks ${ }^{1,2}$, Robert Toth $^{1,2}$, and Anant Madabhushi ${ }^{2}$
}

\begin{abstract}
MRI-guided laser-induced interstitial thermal therapy (LITT) is a form of laser ablation and a potential alternative to craniotomy in treating glioblastoma multiforme (GBM) and epilepsy patients, but its effectiveness has yet to be fully evaluated. One way of assessing short-term treatment of LITT is by evaluating changes in post-treatment MRI as a measure of response. Alignment of pre- and post-LITT MRI in GBM and epilepsy patients via nonrigid registration is necessary to detect subtle localized treatment changes on imaging, which can then be correlated with patient outcome. A popular deformable registration scheme in the context of brain imaging is Thirion's Demons algorithm, but its flexibility often introduces artifacts without physical significance, which has conventionally been corrected by Gaussian smoothing of the deformation field. In order to prevent such artifacts, we instead present the Anisotropic smoothing regularizer (AnSR) which utilizes edge-detection and denoising within the Demons framework to regularize the deformation field at each iteration of the registration more aggressively in regions of homogeneously oriented displacements while simultaneously regularizing less aggressively in areas containing heterogeneous local deformation and tissue interfaces. In contrast, the conventional Gaussian smoothing regularizer (GaSR) uniformly averages over the entire deformation field, without carefully accounting for transitions across tissue boundaries and local displacements in the deformation field. In this work we employ AnSR within the Demons algorithm and perform pairwise registration on $2 \mathrm{D}$ synthetic brain MRI with and without noise after inducing a deformation that models shrinkage of the target region expected from LITT. We also applied Demons with AnSR for registering clinical T1-weighted MRI for one epilepsy and one GBM patient pre- and post-LITT. Our results demonstrate that by maintaining select displacements in the deformation field, AnSR outperforms both GaSR and no regularizer (NoR) in terms of normalized sum of squared differences (NSSD) with values such as $\mathbf{0 . 7 4 3 , 0 . 8 0 7}$, and 1.000, respectively, for GBM.
\end{abstract}

\section{INTRODUCTION}

Laser-induced interstitial thermal therapy (LITT) is a novel MRI-guided therapy to treat brain tumors and epileptogenic

\footnotetext{
* Research reported in this publication was supported by the Department of Defense under award numbers W81XWH-12-PCRP-PTA and W81XWH-11-1-0179; National Cancer Institute of the National Institutes of Health under award numbers R01CA136535-01, R01CA140772-01, and R21CA167811-01; the National Institute of Biomedical Imaging and Bioengineering of the National Institutes of Health under award number R43EB015199-01; the National Science Foundation under award number IIP-1248316; the QED award from the University City Science Center and Rutgers University. The content is solely the responsibility of the authors and does not necessarily represent the official views of the National Institutes of Health.

${ }^{1}$ Department of Biomedical Engineering, Rutgers University, Piscataway, NJ 08854, USA

${ }^{2}$ Department of Biomedical Engineering, Case Western Reserve University, Cleveland, $\mathrm{OH} 44106$, USA

${ }^{3}$ Department of Neurosurgery, University of Medicine and Dentistry of New Jersey, Newark, NJ 07103, USA
}

foci [1], [2]. While the success of LITT has not yet been thoroughly assessed, short term patient response may be evaluated by identifying post-LITT changes in MRI markers. Because LITT consists of exposing the lesion to focused laser energy for target precision, nonrigid registration algorithms aid in detecting detailed and subtle imaging related changes specific to the treatment location by aligning pre- and postLITT images. Therefore, the registration may help identify tissue changes at the LITT site and hence provide accurate prognostic information.

Some popular algorithms are B-splines Free Form Deformation (FFD) [3] and physical partial differential equation (PDE)-based algorithms [4]. Unlike FFD, Thirion's Demons algorithm does not require a user-defined grid of control points but rather captures local deformations at any pixel location. By operating directly on image intensities and gradients, Demons algorithm is also more computationally efficient than registration schemes which approximate solutions to PDEs. Despite these strengths, artifacts may be introduced when the registration reaches an unsmooth deformation field resulting in an anatomically implausible transform. Therefore, the Gaussian smoothing regularizer (GaSR) is applied to restrict locally varying degrees of displacements, thus eliminating unnatural deformations [6].

The disadvantage of GaSR is that it operates in an isotropic fashion and therefore does not preserve local deformations which tend to occur at tissue boundaries [7], [8]. These details are crucial to aligning images with Demons algorithm, especially because the displacements are dependent on edge information, namely the gradient of the fixed image. Lack of such information may in turn weaken the ability of the registration algorithm to fully capture more subtle changes that manifest within sites of localized treatment. When the deformation field is characterized by abrupt changes, a locally adaptive regularizer is required to preserve discontinuities along the boundaries while creating smooth deformations in homogeneous regions.

Cahill et al. [6] presented the convolution-based locally adaptive curvature regularizer for Demons algorithm by approximating the solution to a set of diffusion PDEs [6]. However, its performance was demonstrated to smooth according to the curvature of a vector field. Without the presence of abrupt discontinuities, it is unclear how well the locally adaptive curvature regularizer will be able to capture subtle changes involved in tumor ablation. Similarly, Risholm et al. [7] recognized that registering pre- and post-surgery images using Demons algorithm required discontinuities in the deformation field to signify tissue removal. They adopted the 
classic anisotropic diffusion technique originally proposed by Perona and Malik [9]. The original equation was modified by incorporating strain tensors to model tissue changes before smoothing the deformation field. However, this requires prior knowledge of how imaging markers behave at specific sites, so the use of directional tensors is impractical for preserving subtle displacements whose behavior is still undetermined.

In this work, we present a novel locally adaptive regularizer called the Anisotropic smoothing regularizer (AnSR) that is integrated into Demons algorithm that performs locally varied smoothing on the deformation field. AnSR performs aggressive smoothing in only areas containing homogeneous deformations while performing less aggressive smoothing at or near abrupt local deformations and tissue boundaries. Without imposing any directional constraints such as those found in [7], AnSR aligns images containing slight deformations which may be too subtle to be captured by GaSR or possibly the locally adaptive curvature regularizer [6]. We demonstrate that AnSR is able to capture subtle deformations induced by LITT while smoothing out artifacts inadvertently introduced by the registration algorithm within large homogeneous regions of the deformation field.

We apply Demons algorithm to a pair of 2D synthetic MRI brain images to compare the performances of no regularizer (NoR), GaSR, and AnSR in image alignment. In addition, we evaluate Demons with the three regularization types on preand post-LITT images for an epilepsy patient and a GBM patient.

\section{METHODOLOGY}

\section{A. Thirion's Demons algorithm}

The Demons algorithm registers a pair of images. At each iteration $n$, the difference metric $D^{n}(c)$ is calculated between the fixed image $f(c)$ and moving image $m^{n}(c)$ based on intensity values, for all $c \in C$, where $c$ is pixel location and $C$ is the entire image:

$$
D^{n}(c)=\frac{\left(f(c)-m^{n}(c)\right) \nabla f}{\|\nabla f\|^{2}+\left(f(c)-m^{n}(c)\right)^{2}} .
$$

The individual difference vectors calculated from each pixel in $D^{n}(c)$ can be used directly as displacements in the deformation field: $U^{n}(c)=D^{n}(c)$, for all $c \in C \cdot U^{n}(c)$ can then be applied to $m^{n}(c)$ to align onto $f(c)$ such that $m^{n}\left(c+U^{n}(c)\right)$ approaches $f(c)$ as $n \rightarrow \infty$.

However, using $D^{n}(c)$ directly as the deformation field yields physically implausible deformations that are no longer anatomically meaningful. Therefore, a regularizer is applied to reduce such unnatural displacements for improved alignment. Instead of applying the $D^{n}(c)$ directly by equating it to $U^{n}(c)$ for the current iteration $n$, the deformation field from a previous iteration or a separate calculation denoted generally by $U^{n *}(c)$ is added to $D^{n}(c)$ and smoothed by a regularizer $S$ such that

$$
\hat{U}^{n}(c)=S\left(D^{n}(c)+U^{n *}(c)\right)
$$

before $m^{n}(c)$ is replaced by $m^{n}\left(c+\hat{U}^{n}(c)\right)$.
TABLE I: Demons Algorithm with AnSR pseudocode

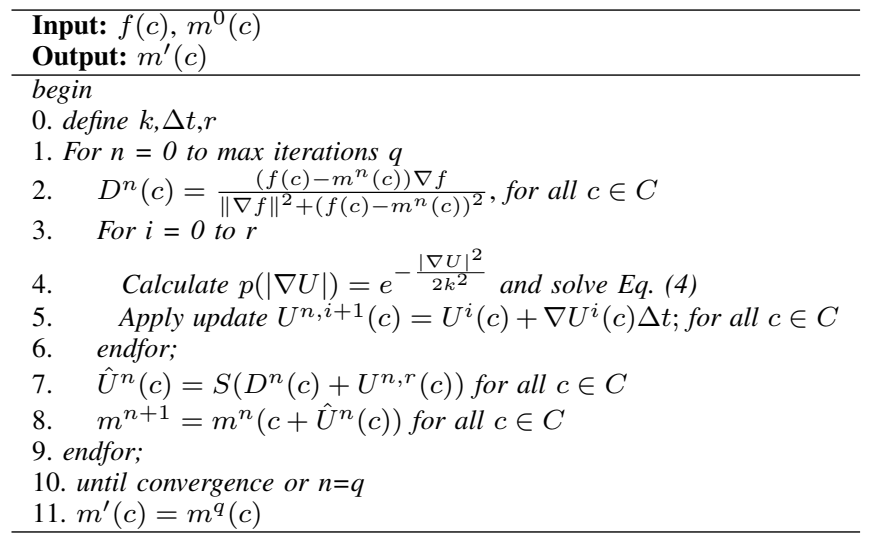

\section{B. Smoothing regularizers}

1) Gaussian smoothing regularizer (GaSR): GaSR is a 2D Gaussian kernel whose size is the standard deviation $\sigma$ defined by the user. This regularizer is isotropic, meaning that as it moves across the entire image it uniformly averages the deformations across all dimensions. The larger the $\sigma$, the more aggressive the smoothing. GaSR yields a smooth deformation field $\hat{U}^{n}(c)=S\left(D^{n}(c)+U^{n-1}(c), \sigma\right)$ before $m^{n}(c)$ is aligned to $f(c)$ to yield a final registered image $m^{\prime}(c)$ that would otherwise contain artifacts due to implausible deformations without regularization. Note that here $U^{n *}(c)=U^{n-1}(c)$ in Eq. (2).

2) Anisotropic smoothing regularizer (AnSR): AnSR uses finite forward differences to iteratively approximate a solution to the anisotropic diffusion equation, a partial differential equation initially presented in [9]:

$$
\frac{\partial U(x, y, t)}{\partial t}=\nabla \cdot \nabla U(x, y, t)
$$

where $\nabla$ represents the image gradient. By default, the solution is the deformation field smoothed by GaSR, but a conductance function $p(|\nabla U|)=e^{-\frac{|\nabla U|^{2}}{2 k^{2}}}$ was introduced to reduce smoothing in areas with a large gradient magnitude, resulting in

$$
\frac{\partial U(x, y, t)}{\partial t}=\nabla \cdot p(|\nabla U|) \nabla U .
$$

Thus, AnSR is superior to isotropic regularizers such as GaSR in that it is locally adaptive. As a result, the deformation field contains more natural displacements without losing important details along the boundaries of anatomical structures. The user is able to define: 1) the conductance parameter $k$ which scales the aggressiveness of smoothing by $p(|\nabla U|), 2)$ the time step $\Delta t$ of the finite forward difference equation to control the size of the filter, and 3 ) the number of smoothing iterations $r$ :

$$
\hat{U}^{n}(c)=S\left(D^{n}(c)+U^{n, r}(c)\right),
$$

where the smoothed deformation field $\hat{U}^{n, r}(c)$ is the approximate solution to Eq. (4) using the chosen parameters and $U^{n *}(c)=\hat{U}^{n, r}(c)$ in Eq. (2). The algorithm presented in 


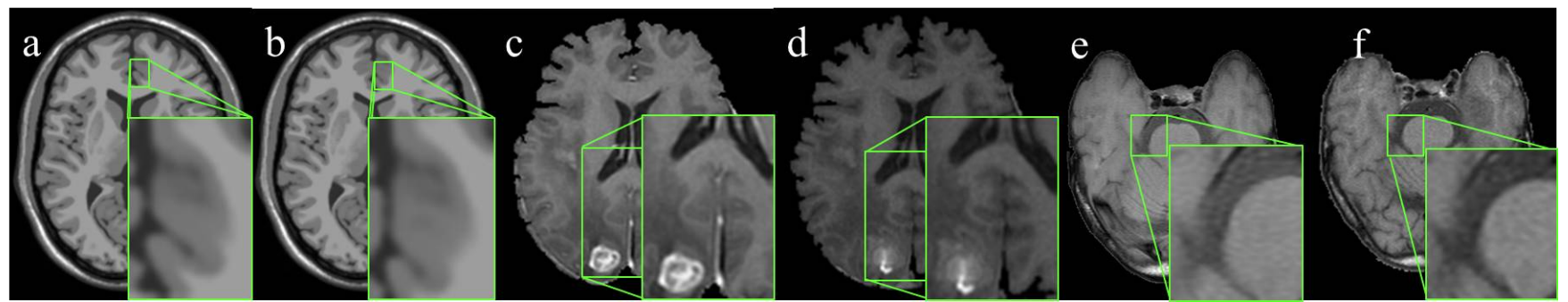

Fig. 1: The fixed and moving images with selected regions of interest (ROI)s used to evaluate performance. Figure 1(a) shows the original BrainWeb image on which an artificial deformation was induced to generate the moving image Figure 1(b). The pre- and post-LITT GBM images are presented in Figure 1(c),(d), respectively. Figure 1(e),(f) show the pre- and post-LITT epilepsy images, respectively.

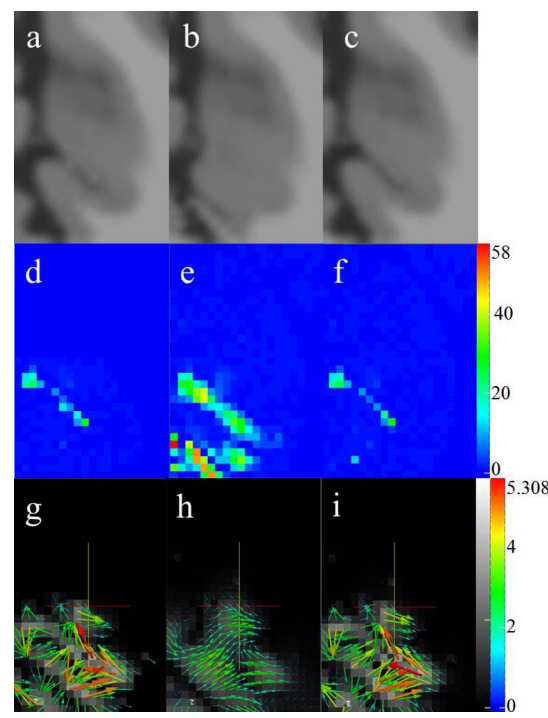

Fig. 2: The results from the synthetic experiment on pre- and post-LITT MRI. Figure 2(a),(b),(c) show the registered images using Demons with NoR, GaSR, and AnSR, respectively. Figure 2(d),(e),(f) present the corresponding difference between the registered images and the fixed image and (g),(h),(i) show the corresponding deformation field magnitudes.

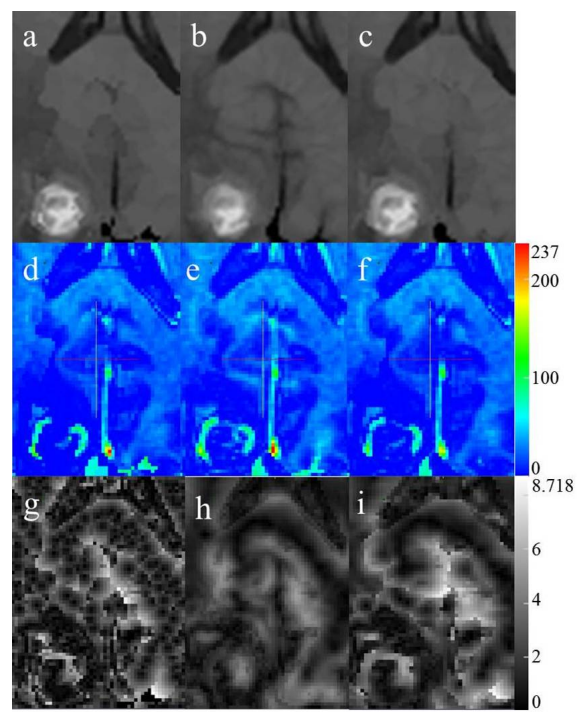

Fig. 3: The results from the clinical GBM experiment on pre- and post-LITT MRI. Figure 3(a),(b),(c) show the registered images using Demons with NoR, GaSR, and AnSR, respectively. Figure 3(d),(e),(f) present the corresponding difference between the registered images and the fixed
image and (g),(h),(i) show the corresponding deformation field magnitudes, where the arrows have
been removed for clarity.

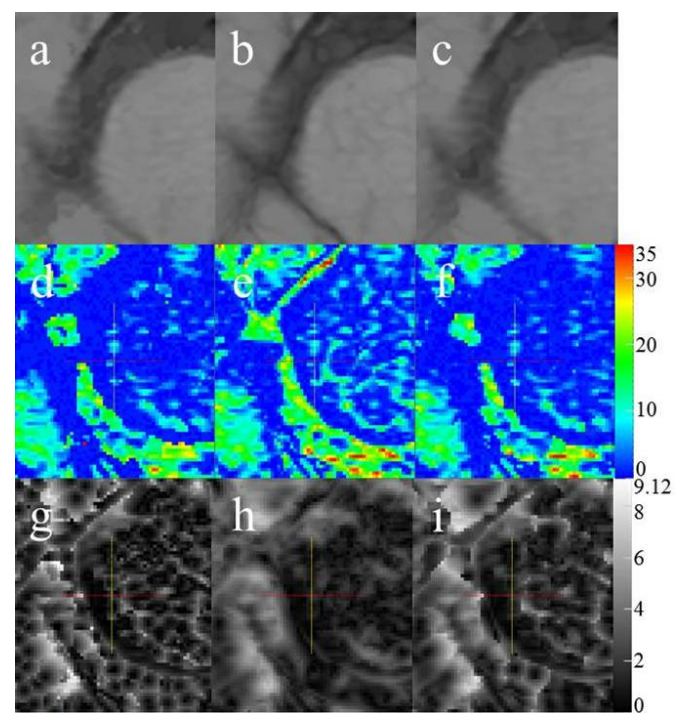

Fig. 4: The results from the clinical epilepsy experiment on pre- and post-LITT MRI. Figure 4(a),(b),(c) show the registered images using Demons with NoR, GaSR, and AnSR, respectively. Figure 4(d),(e),(f) present the corresponding difference between the registered images and the fixed image and (g),(h),(i) show the corresponding deformation field magnitudes, where the arrows have been removed for clarity.
Table I demonstrates how AnSR is incorporated into Demons algorithm.

\section{EXPERIMENTAL RESULTS AND DISCUSSION}

\section{A. Dataset description}

Demons regularized with AnSR was validated on a series of 1) synthetic experiments and 2) clinical experiments on brain MRI. The first study used 2D synthetic brain MRI downloaded from the McBIC MNI BrainWeb database at 181 x 217 with $1 \mathrm{~mm} /$ pixel resolution. A deformation was induced to simulate ablation of tissue on one image and the experiment was repeated with $5 \%$ noise. The second study registered 512 × 512 T1-weighted brain MRI taken pre- and post-LITT to treat an epilepsy patient and a GBM patient. The skull and background were masked out and an initial alignment between the pre- and post-LITT images was performed using affine registration.

\section{B. Implementation and Performance Measures}

The algorithms were programmed using Insight Segmentation and Registration Toolkit (ITK) with images visualized in Paraview. For all registration experiments, the Demons algorithm was employed with NoR, GaSR ( $\sigma=2.0)$, and AnSR $(k=2.0, \Delta t=0.067, n=5)$. The parameters for all registration experiments were determined empirically and kept consistent across all studies. Performance was evaluated within particular regions of interest (ROIs) that exhibited

TABLE II: Description of the datasets used for evaluation

\begin{tabular}{|l|l|l|}
\hline Type & Experiment & Objective \\
\hline Synthetic & $\begin{array}{l}\text { Deformation (no } \\
\text { noise) }\end{array}$ & Recover original deformation \\
\hline Synthetic & $\begin{array}{l}\text { Deformation } \\
\text { (noise) }\end{array}$ & $\begin{array}{l}\text { Recover original deformation in the } \\
\text { presence of noise }\end{array}$ \\
\hline Clinical & GBM & Register pre- and post-LITT MRI \\
\hline Clinical & Epilepsy & Register pre- and post-LITT MRI \\
\hline
\end{tabular}


the need for locally adaptive regularization, such as lesion sites and tissue interfaces. Quantitative results were obtained by calculating the registration error via normalized sum of squared difference (NSSD) of image intensities, which is defined by the SSD normalized by the maximum SSD among the three types of regularization for each study.

\section{Co-registering synthetic brain images}

A synthetic deformation was induced on the fixed image (Figure 1(a)) to generate the moving image (Figure 1(b)) in order to model the shrinkage in the tumor as expected from LITT, and the three types of Demons algorithm were performed (NoR, GaSR, AnSR). The results in Figure 2 concentrate on the ROI to display subtle differences in registered images (Figure 2(a),(b),(c)) in registration error (Figure 2(d),(e),(f)) arising from deformations that are only appreciable locally. In Figure 2, AnSR helps preserve deformation on the boundaries and tissue interfaces, where the local deformations are not smoothed over compared to GaSR (Figure 2(h)). On the other hand, NoR results in a large number of spurious changes being identified on the difference image. Therefore, the corresponding deformation fields in Figure 2(g),(h),(i) demonstrate the value of the selective smoothing with more aggressive smoothing of the deformation field within the larger homogeneous regions of the deformation field and more conservative smoothing on the tissue interfaces. According to the NSSD values calculated on the ROI (Figure 5), AnSR and NoR result in very similar error magnitudes and are much less than that of GaSR. Adding 5\% noise to this experiment increased the error for all three regularizers, but did not change the relative rankings.

\section{Co-registering pre- and post-LITT brain images}

After skull-stripping and affine pre-alignment, the postLITT images for epilepsy and GBM were registered to their respective pre-LITT images. Figure 1(c),(d) display the pre- and post-LITT MRI for the GBM experiment. Note that the lesion is located in the lower left corner. After performing Demons registration, the registered images in Figure 3(a),(b),(c) show that with NoR, artifacts emerge as discrete patches of identical intensity, while these artifacts are not apparent with AnSR and GaSR. The difference in the ROI (Figure 3(d)) is less than that of AnSR and GaSR (Figure 3(e),(f)) and is an example of artifacts introduced by NoR. On the other hand, GaSR caused displacements (Figure 3(h)) that were too smooth and failed to recover the pre-LITT image. However, AnSR best recovered the deformation caused by LITT while reducing the artifacts that would have emerged without regularization. Similarly, the pre- and post-LITT MRI are displayed in Figure 1(e)(f) for the epilepsy experiment with the registered images, difference images, and deformation fields in Figure 4. In the epilepsy results, AnSR also appears to eliminate more artifacts (Figure 4(f)) than with NoR or GaSR (Figure 4(d),(e)) for the same reasons described for the GBM data. As shown in Figure 4(g),(h),(i), AnSR outperforms the other

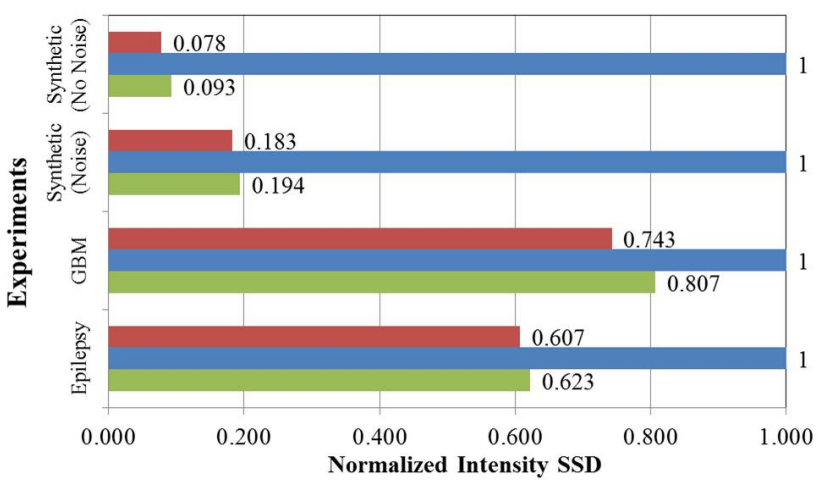

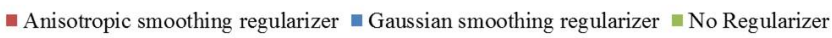

Fig. 5: NSSD values for all experiments comparing the three regularizers.

two methods by: 1) preserving the local displacements at tissue interfaces that would otherwise be blurred by GaSR and 2) avoiding the artifacts that would otherwise occur without regularization. Figure 5 reveals that for both the GBM and epilepsy datasets, within localized regions, NSSD was lower for AnSR compared to GaSR and NoR.

\section{CONCLUDING REMARKS}

LITT is a recent form of laser ablation for brain lesions and epilepsy, but its effect on tissue is poorly understood. In this work we presented the Anisotropic smoothing regularizer (AnSR) in the context of Demons, which may help effectively align of pre- and post-LITT MRI to accurately capture local tissue changes. On both synthetic and clinical epilepsy and GBM MRI, AnSR was shown to outperform both Gaussian smoothing regularizer (GaSR) and no regularization (NoR). Future work will involve additional extensive evaluation of AnSR on more patient data.

\section{REFERENCES}

[1] A.Carpentier et al., "MR-guided laser-induced thermal therapy (LITT) for recurrent glioblastomas," Lasers in Surgery and Medicine, vol. 44, pp. 36168, 2012.

[2] J.F.Tellez-Zenteno et al., "Surgical outcomes in lesional and nonlesional epilepsy: A systematic review and meta-analysis," Epilepsy Research, vol. 89, pp. 31018, 2010.

[3] D. Rueckert et al., "Nonrigid Registration Using Free-Form Deformations: Application to Breast MR Images," vol. 18, no. 8, pp. 712-721, 1999.

[4] G. E. Christensen et al., "Deformable Templates Using Large Deformation Kinematics," vol. 5, no. 10, pp. 1435-1447, 1996.

[5] J.-P. Thirion, "Image matching as a diffusion process: an analogy with Maxwell's demons," in Medical Image Analysis, vol. 2, no. 3, pp. 243260, 1998.

[6] N. D. Cahill et al., "A Demons Algorithm for Image Registration with Locally Adaptive Regularization," in Proc. MICCAI 2009, LNCS 5761, 2009.

[7] P. Risholm et al., "A Non-rigid Registration Framework That Accommodates Resection and Retraction," in Inf. Process Med. Imaging, vol. 21, pp. 447-458, 2009.

[8] A. Tristn-Vega and S. Aja-Fernndez, "Fuzzy regularisation of deformation fields in image registration," in Proc. IPMU 2008, pp. 1223-30, 2008.

[9] P. Perona and J. Malik, "Scale-Space and Edge Detection Using Anisotropic Diffusion," in IEEE Trans. Pattern Analysis and Machine Intelligence, vol. 12, no. 7, pp. 629-639, 1990. 